\title{
Communicating to Tourists During and Post-Covid-19: What Do They Want (Need) to Hear?
}

\author{
Laura Zizka ${ }^{(\bowtie)}$ (D), Meng-Mei Chen (D), Effie Zhang, \\ and Amandine Favre \\ Ecole Hoteliere de Lausanne//HES-SO University of Applied Sciences \\ and Arts Western Switzerland, Lausanne, Switzerland \\ \{Laura. ZIZKA, Maggie. CHEN-HOLLERAN, Effie. ZHANG2, \\ Amandine.FAVRE\}@ehl.ch
}

\begin{abstract}
Swiss tourism relies heavily on international clientele to book rooms and purchase goods and services. However, from March to June 2020, due to the COVID-19 pandemic, travel and subsequent bookings slowed and, in some cases, stopped altogether. Based predominantly on Situational Crisis Communication Theory (SCCT), this paper investigates Swiss hotels' messages on their official Facebook pages and the SCCT strategies they employed during this crisis. The findings from 48 independent four- and five-star hotels show that the Victimage strategy was the most often employed when communicating about the COVID-19 pandemic in general. Further, most hotels published positive messages during this period (68\%) and strongly emphasized their roles as victims at this time. Only $5 \%$ of the messages posted were negative messages. Hotels 'blamed' the government and the sanitary measures for their closing or reduced services. The findings presented here contribute to the literature by offering a pattern of crisis responses from Swiss hotels in the early period of the pandemic. These results are currently being updated with the messages communicated in the 12 months since the beginning of this study. The findings of this crisis communication during an early stage of the pandemic will be used to make concrete recommendations for the strategies that should be implemented in the future if the COVID-19 crisis continues or when faced with other crises.
\end{abstract}

Keywords: Swiss hotels $\cdot$ COVID-19 $\cdot$ Facebook $\cdot$ Situational Crisis Communication Theory $(\mathrm{SCCT}) \cdot$ Crisis communication strategies

\section{Introduction}

Tourism in Switzerland is a lucrative business. The tourism industry is one of the largest export industries in Switzerland with $4.4 \%$ of export revenue [1], providing many jobs to the local communities and contributing significantly to the local economy in general. According to the 2019 official statistics, Swiss tourism includes 28985 hotel and restaurant establishments that generate $\mathrm{CHF} 44.7$ billion in total revenue, CHF 16.6 billion from foreign tourists in Switzerland [2]. 
The 2019 tourist season was a profitable time for Swiss tourism. The expectations for 2020 were equally positive until late December 2019, when news of the COVID-19 pandemic and its potential to spread globally was shared.

As word of the COVID-19 pandemic spread, the Swiss tourism industry experts began making ominous predictions regarding the 2020 tourism season, and early statistics confirmed those predictions. According to a press release from the official Swiss Federal Statistical Office (FSO) on Feb. 2, 2021- "In 2020, the Swiss hotel sector registered 23.7 million overnight stays. This represents a fall of $40.0 \%$ ( -15.8 million overnight stays) compared with 2019. The exceptional context of COVID-19 explains this unprecedented fall at a level not seen since the end of the 1950s. Foreign demand registered 7.3 million overnight stays, i.e., a decline of $66.1 \%$ (-14.3 million). Swiss demand fell less drastically $(-8.6 \% /-1.5$ million) to 16.4 million overnight stays." (para.1) [3].

Previous studies have examined crises in the hospitality industry, and Swiss hotels have remained relatively unscathed from previous crises. Nonetheless, with the COVID-19 pandemic, Swiss tourism was significantly affected. In the period examined in this study (March through June 2020), Swiss tourism saw foreign demand fall by $68 \%$ in March and overnight stays by Swiss guests by 55\% [3]. The number of Swiss guests declined further in April 2020 (by -86\%) and rose slightly in May $2020(-54 \%)$ [3]. With the relaxation of some of the COVID-19 restrictions, domestic rose slightly to only $23 \%$ less than the previous year's statistics [3].

This study aims to investigate the messages that Swiss hoteliers provided to their clients on their official Facebook pages. As many clients use social media, particularly Facebook, to gather information before traveling, this communication channel was selected as the most appropriate for analyzing the SCCT strategies these hotels employed. The overarching objective is to establish the strategies used and gauge their effectiveness in communicating during a crisis, namely, the COVID-19 pandemic. These results can be used to communicate 'better' when the next crisis hits the hospitality industry.

\section{Literature Review}

\subsection{Crisis and Situational Crisis Communication Theory (SCCT)}

Crises have been defined as unpredictable events that can disrupt an organization's operations, impact their performance and, potentially, generate negative feelings toward the organization leading to reputational damage [4]. While an organization is concerned about its reputation, public safety and keeping customers safe comes first [5]. The COVID-19 pandemic is a crisis where the organization has little to no responsibility or control over the circumstances. This type of crisis is categorized by stakeholders as a Victim crisis, as the organization could do nothing to prevent its occurrence [6]. With this global pandemic, all industries were affected, but some industries fared better than others. For the tourism industry, COVID-19 had significant consequences. In this paper, one part of the broader tourism industry, i.e., hotels, will be examined to gauge how they faced the pandemic. 
According to Coombs Situational Crisis Communication Theory (SCCT), when the organization has been attributed weak responsibility or has been identified as a Victim crisis such as COVID-19, the organization should implement an ethical base response. This base response consists of instructing information (i.e., explaining the crisis to the stakeholders) and adjusting information (i.e., helping stakeholders cope with the crisis) [7]. This is the overarching strategy that should be used in the case of any crisis. However, organizations can choose to employ other strategies as well based on the crisis. For example, they could include SCCT strategies such as ingratiation (i.e., praising stakeholders for efforts they have made), victimage (i.e., confirming that the organization is a victim of this crisis), or reminder (i.e., telling stakeholders the good things you did in the past) [8]. These are part of the crisis communication strategy cluster known as bolster [9]. Other crisis communication strategy clusters include instructing and adjusting (i.e., the base response of all crisis messages), deny, diminish, and rebuild [9]. Depending on the type of crisis, the attribution of responsibility, and the intensifying factors, organizations will choose which response or which combination of responses best fits the crisis at hand.

The research has evolved to include crisis communication strategies consisting of enhancing, scapegoating, and transferring. Enhancing is defined as telling stakeholders about current good works the company is doing) [10]. Scapegoating occurs when companies shift the blame to another group outside of the organization [11]; this is part of the deny cluster [9]. Transferring entails naming a credible third party as part of the crisis response [10]. This paper examines how Swiss independent hotels communicated crisis responses on their official Facebook pages during 3 months in 2020.

\subsection{Crisis Communication in the Hospitality Industry}

Researchers have examined how and what companies communicate with their stakeholders in times of crisis. In the hospitality industry, specifically, researchers have examined the messages posted on social media platforms such as Twitter or Facebook during crises. Tourism organizations are encouraged to use social media to communicate during crises as many tourists turn to social media to seek information about the destination or property [12]. After all, their stakeholders are using social media and often post on their own social media accounts while on vacation [SAME]. However, studies have shown that the hospitality industry, in particular, has not been proactive and does not take advantage of the reach that social media has during crises [8]. Further, some hospitality organizations have given inappropriate responses in times of crisis [11]. The hospitality industry must become less averse to social media and embrace it as a potential channel for crisis communication. As Switzerland is dependent on international travel for their tourism, particularly for hotel occupancy, this study will analyze the crisis communication strategies used on the official Facebook pages and address the following two research questions:

RQ1: What crisis communication strategies were employed most frequently during the first wave of COVID-19 by Swiss hoteliers?

RQ2: What sentiment were the Swiss hoteliers trying to communicate to their clients during this first wave of COVID-19? 


\section{Methodology}

This paper examines the messages during and after the first wave and first confinement of 48 Swiss four- and five-star, independent hotels to analyze the crisis communication strategies they employed during this period. Their messages posted on their official Facebook pages are measured against Situational Crisis Communication Theory (SCCT). Both content analysis and a sentiment analysis of their messages were conducted to establish the strategies they used and the emotion they created in doing so. This paper concludes with the implications of effectively using crisis communication strategies during the COVID-19 pandemic and future crises.

To examine the messages posted on Swiss hotel Facebook pages, a list of 222 independent hotels (four- and five-star independent hotels in Switzerland) was created. Of the original 222 hotels, only 96 had Facebook pages. Of the 96 remaining hotels, those that were part of a group (though not a chain) and hotels that were closed during this period were eliminated. The remaining 48 hotels were used in this study, and a comparison between their Facebook traction in 2019 versus 2020 was made. The Facebook comments were tracked from mid-crisis when many hotels were closed (March 13, 2020) to June 13, 2020, again, when many of the hotels reopened. The same dates were used in 2019 as well. Some hotels were preparing to open their doors when this study was conducted, while others communicated the reopening date. This study analyzes the crisis communication strategies employed by Swiss hotels during this first de-confinement stage.

The research team collected all posts and calculated descriptive statistics, including means and standard deviations. Two research assistants independently coded all comments based on the SCCT strategies and recorded the frequencies. Their results were compared to seek differences. Any differences were discussed until a final agreement was reached. A sentiment analysis was conducted by inserting each post to the sentiment analysis tool available at Monkeylearn.com. For each post, Monkeylearn.com classifies it as positive, negative, or neutral and provides its confidence level. The closer the confidence level is to one, the more reliable the result. The research team recorded the sentiment classification, frequencies, and confidence level.

\section{Results}

\subsection{Descriptive Statistics Results}

The means and standard deviations for the numbers of posts are 23.85 (S.D. 24.46) and 1.98 (S.D. 1.54) for 2019 and 2020, respectively. The two tail t-test result (t $(47)=6.296, p=0.000)$ confirmed the significant difference between 2019 and 2020 . While the literature suggests that communication during a crisis should be frequent, timely, and consistent [7], the lack of communication from Swiss hoteliers seems to indicate the opposite. They posted quite infrequently, particularly in comparison with the same period in 2019. One explanation could be that the hotel business is quite competitive; thus, hotels post their special offers to attract clients during their busiest seasons. In the case of COVID-19, there was nothing new to post and nothing to offer. 
Further, international tourists who could not travel due to sanitary restrictions, quarantines, or lockdowns would not search for hotel information on Facebook. The typical exchanges on social media, i.e., travel stories or photos, were not posted during this period.

\subsection{Hotel Official Facebook Comments - Frequency of SCCT Strategies}

The Swiss hotels examined in this study are located in various regions in Switzerland. Thus, many of the messages are communicated in the local language. Switzerland has four official languages, French, German, Italian, and Romansch, but, as an international travel destination, Swiss hotels are obliged to provide messages in English as well. In this study, we focused on all the messages communicated in English. On Table 1, the frequency of the types of SCCT strategies employed by the hotels are analyzed. The only SCCT strategy that was not employed was apology; this result could be expected as all hotels were victims of this crisis.

Table 1. Frequency of SCCT strategies employed by Swiss hotels

\begin{tabular}{l|c|c}
\hline Strategies & Number of times employed & Frequency \\
\hline Scapegoat & 11 & $12 \%$ \\
\hline Justification & 8 & $9 \%$ \\
\hline Apology & 0 & $0 \%$ \\
\hline Compensation & 7 & $8 \%$ \\
\hline Endorsement & 2 & $2 \%$ \\
\hline Enhancing & 5 & $5 \%$ \\
\hline Ingratiation & 10 & $11 \%$ \\
\hline Reminder & 3 & $3 \%$ \\
\hline Victimage & 46 & $49 \%$ \\
\hline Transferring & 1 & $1 \%$ \\
\hline Total & 93 & $100 \%$ \\
\hline
\end{tabular}

From the results, only one strategy, victimage, was used in $49 \%$ of posts communicating about the Covid-19 situation. All the other strategies were employed less than $12 \%$ of the time, such as scapegoat (12\%), ingratiation (11\%), justification (9\%), and compensation (8\%), enhancing (5\%), reminder (3\%), endorsement $(2 \%)$, and transferring $(1 \%)$. To further investigate these strategies, a sentiment analysis was conducted with all the posts. Table 2 displays those results. As seen in the literature, ingratiation and victimage derive from the bolster crisis communication cluster.

\subsection{Hotel Facebook Comments and Sentiment Analysis}

The 93 messages on Facebook were also examined through sentiment analysis. As seen on Table 2, the vast majority of the messages posted were classified as positive messages. Nonetheless, there were examples of positive, negative, and neutral in the data. 
The confidence levels for positive messages ranged from .4 to .99 indicating a strong relationship between the words and the sentiment as the closer to one, the more reliable the result. For negative messages, the confidence levels ranged from .56 to .80 and, for neutral messages, from .40 to .94 .

Table 2. Sentiment analysis and frequency of communication strategies

\begin{tabular}{l|r|r|l|l|l|l|r|r}
\hline Sentiment & \multicolumn{2}{|l|}{ Positive } & \multicolumn{2}{l}{ Negative } & \multicolumn{2}{l}{ Neutral } & \multicolumn{2}{l}{ Total } \\
\hline Strategies & $\#$ & In $\%$ & $\#$ & In $\%$ & $\#$ & In $\%$ & $\#$ & In $\%$ \\
\hline Scapegoat & 0 & $0 \%$ & 1 & $1 \%$ & 10 & $11 \%$ & 11 & $12 \%$ \\
\hline Justification & 3 & $3 \%$ & 1 & $1 \%$ & 4 & $4 \%$ & 8 & $9 \%$ \\
\hline Apology & 0 & $0 \%$ & 0 & $0 \%$ & 0 & $0 \%$ & 0 & $0 \%$ \\
\hline Compensation & 3 & $3 \%$ & 0 & $0 \%$ & 4 & $4 \%$ & 7 & $8 \%$ \\
\hline Endorsement & 1 & $1 \%$ & 0 & $0 \%$ & 1 & $1 \%$ & 2 & $2 \%$ \\
\hline Enhancing & 2 & $2 \%$ & 1 & $1 \%$ & 2 & $2 \%$ & 5 & $5 \%$ \\
\hline Ingratiation & 9 & $10 \%$ & 1 & $1 \%$ & 0 & $0 \%$ & 10 & $11 \%$ \\
\hline Reminder & 2 & $2 \%$ & 0 & $0 \%$ & 1 & $1 \%$ & 3 & $3 \%$ \\
\hline Victimage & 43 & $46 \%$ & 1 & $1 \%$ & 2 & $2 \%$ & 46 & $49 \%$ \\
\hline Transferring & 0 & $0 \%$ & 0 & $0 \%$ & 1 & $1 \%$ & 1 & $1 \%$ \\
\hline Total & 63 & $68 \%$ & 5 & $5 \%$ & 25 & $27 \%$ & 93 & $100 \%$ \\
\hline
\end{tabular}

Overall, comments classified as positive were the most frequent in the study at $68 \%$, followed by neutral $(27 \%)$ and negative $(5 \%)$. This finding indicates that under difficult times, hoteliers still wanted to create positive sentiments. Neutral responses were relatively common which could be explained by the need to give 'distressing' information while still holding a positive attitude. Only five messages were categorized with negative sentiment. Nonetheless, these results can be explained by the SCCT strategy adopted.

Based on Table 1, victimage and scapegoat are the most adopted strategies. Table 2 demonstrated that different strategies could be applied in positive, negative, or neutral tones. Victimage was the dominant strategy employed by Swiss hotels and was primarily executed in positive tones but could be in negative, or neutral tones. For example, a positive sentiment analysis phrase for victimage read: 'We wish you a lot of energy for the coming weeks and are already looking forward to welcoming you again in the summer' (confidence level $=.67$ ). A neutral sentiment analysis of victimage stated: 'The hotel is closed at this due to Corona measures' (confidence level $=.50$ ). The negative example of victimage was: 'Finally it starts again today Wednesday, and we will see you again after a long break' (confidence level $=.56$ ).

For comments with negative sentiment analysis, five SCCT strategies were used equally: Justification, victimage, ingratiation, enhancing, and scapegoat. Nonetheless, the negative category had the least comments (one per category), which could explain this even spread. Justification, victimage, and scapegoat may be explained by the need 
to 'blame' someone outside of themselves or to justify the measures they have taken against their will. As these decisions were imposed on the hotels, they may have felt obliged to communicate this to their clients. The only other strategies used with negative sentiment analysis were enhancing and ingratiation.

For neutral sentiment analysis comments, eight SCCT strategies were employed. The least frequent strategies were reminder, endorsement, and transferring (once each), enhancing and victimage (twice each), and justification and compensation (four times each). Scapegoat was the most commonly used strategy (10 times). The latter could be explained by the hoteliers' need to blame someone else for their situation; it appeared simpler to identify an enemy, i.e., COVID-19, than to admit a fault that was clearly not their own.

By conducting both the SCCT strategy analysis and sentiment analysis, this research advances the SCCT research in the hospitality industry to another level.

As shown in Table 2, the SCCT provides a general guideline, but these strategies can be executed in different tones. For example, victimage, justification, and enhancing appeared in positive, negative, and neutral sentiment analysis results. Practitioners are encouraged to explore the different sentiment tones when implementing the SCCT strategies.

\section{Discussion}

As mentioned in the literature review, The COVID-19 pandemic was not a unique crisis affecting an individual property or region; instead, this crisis affected all hotels and regions equally. There was minimal responsibility attribution allocated to the hotels; thus, their response could be justifiably minimal as well. Many establishments could have posted only a base response, i.e., instructing and adjusting information without communicating further. In some cases, hoteliers did not need to communicate about the sanitary measures if their establishment was closed or had only limited business; thus, even the base response was unnecessary. Nonetheless, from this study, it can be seen that many Swiss hoteliers chose to offer more than the base response. Further, they attempted to communicate positively despite the dire circumstances and immediate bleak season in sight.

RQ1: What crisis communication strategies were employed most frequently during the first wave of COVID-19 by Swiss hoteliers?

When they communicated via Facebook, Swiss hoteliers predominantly employed the victimage strategy, i.e., reminding customers that the hotels are victims of this crisis as well as the clients. In this way, it is a shared victim status that could, in theory, bring customers and hoteliers together for a common cause. While seemingly contradictory, a victimage strategy is not negative in the case of a crisis; instead, it shows that the stakeholders do not attribute blame to the company, i.e., the company could do nothing to avoid this crisis. Of the other strategies, scapegoat, ingratiation, justification, and compensation were used in fewer than $12 \%$ of the messages. No apologies were offered as the pandemic was not a crisis that was in their control.

RQ2: What sentiment were the Swiss hoteliers trying to communicate to their clients during this first wave of COVID-19? 
As seen from the sentiment analysis, Swiss hoteliers preferred to focus on positive messages to convince the clientele that the opportunity to return to their properties had finally arrived. These messages did not inform the guests that the experience could potentially be different from previous stays; instead, the messages focused on getting the clients back. Swiss hoteliers did not post daily updates on the COVID-19 pandemic nor the status of their hotels. While they may have missed an opportunity to show compassion or empathy, as the literature advises, their strategy revolved around the better days to come.

\section{Conclusion/Implications}

Coombs outlines the importance of preparing strategic messages that align with a company's crisis and applying the appropriate SCCT strategies to communicate these key messages. Based on the SCCT theory, crisis communication teams can brainstorm potential crises for their industry and proactively prepare crisis communication templates for the different communication channels they use. The COVID-19 pandemic was the latest in a long line of health crises that the hospitality industry has endured. However, the extent of the COVID-19 pandemic and its long-lasting effect on the industry has yet to be seen. While some hotels have reopened, closed, and opened again, others have suffered lay-offs and, in some cases, closures.

The COVID-19 pandemic placed all hotels (and their guests) in the position of the victim. This could explain why the victimage strategy was employed in their Facebook posts. Swiss hoteliers preferred to state the facts, i.e., "we are closed due to COVID19", followed by frequent communication about the ever-changing COVID-19 status or regulations, i.e., "guests must wear masks in the public areas" or "hand sanitizers are available in all public spaces." With the vaccine and the falling number of new COVID-19 cases, hoteliers need to take strategic decisions on how to reopen safely and sustainably without putting themselves, their guest, or their reputations at risk.

As the sanitary conditions improve and more guests and employees are vaccinated, Swiss hotels can begin reflecting on how well they dealt with the COVID-19 crisis and communicated about it during this unprecedented crisis. While many hotels reduced their Facebook posts, there may have been a better strategy to use. These hotels could have opened a dialogue with their customers to entice them to return once the situation improved. Swiss hotels could have proactively promoted next season's opportunities or offers instead of waiting until they were open.

\section{Limitations/Future Studies}

This study had several limitations. Firstly, the hotels were limited to four- and five-star Swiss independent properties that posted messages from March to June 2020 on their official Facebook page. Future studies could evaluate other social media platforms or other periods. Secondly, this study focused on Swiss hotels. Comparative studies against other countries could be preferable in the future to identify best practices in communicating about a crisis on an industry level. Thirdly, the messages in this study derived uniquely from the hotels' official Facebook pages. A further study should investigate the comments posted by the other stakeholders as well. Finally, while the 
SCCT strategies and their sentiments were analyzed in this paper, there was a lack of discussion on the influence of these messages on their clients. A future study could examine the relationship of these strategies with the public's response or emotions.

When this ENTER2022 conference occurs in January 2022, the tourism industry will have opened, closed, and opened again, with restrictions being relaxed only to return to lockdown. Now that the base of SCCT strategies has been established during this time frame, a study is currently being undertaken to analyze the messages posted on social media for the nine months that followed. Examining a full year of crisis messages will inform tourism professionals of the best practices to employ when a future crisis arrives. In the end, a complete picture of what Swiss hoteliers communicated through the various phases of the COVID-19 pandemic will be examined to gauge which strategies were used and their overall effectiveness.

\section{References}

1. Swiss tourism in figures 2018 (2019) Structure and industry data. https://www.stv-fst.ch/ sites/default/files/2019-07/STiZ_2018_EN_Web.pdf

2. Swiss hotel industry enjoyed record year in 2019. https://www.swissinfo.ch/eng/tourism_ swiss-hotel-industry-enjoyed-record-year-in-2019/45583872

3. Record drop in overnight hotel stays in Switzerland in 2020 (2021). https://www.bfs.admin. $\mathrm{ch} / \mathrm{bfs} / \mathrm{en} / \mathrm{home} /$ statistics/tourism.assetdetail.16044296.html

4. Coombs WT, Holladay SJ (2002) Helping crisis managers protect reputational assets: initial tests of the situational crisis communication theory. Manag Commun Q 16:165-186

5. Coombs WT (2007) Protecting organization reputations during a crisis: the development and application of situational crisis communication theory. Corp Reput Rev 10(3):163-176

6. Claeys A-S, Cauberghe V, Vyncke P (2010) Restoring reputations in times of crisis: an experimental study of the situational crisis communication theory and the moderating effects of the locus of control. Pub Relat Rev 36:256-262

7. Coombs WT (1995) Choosing the right words: the development of guidelines for the selection of the 'appropriate' crisis response strategies. Manag Commun Q 8:447-476

8. Barbe D, Pennington-Gray L (2018) Using situational crisis communication theory to understand Orlando hotels: Twitter response to three crises in the summer of 2016. J Hosp Tour Insights 1:1-18

9. Scheiwiller S, Zizka L (2021) Strategic responses by European airlines to the Covid-19 pandemic: a soft landing or a turbulent ride? J Air Transp Manag 95:1-10

10. Cheng Y (2018) How social media is changing crisis communication strategies: evidence from the updated literature. J Conting Crisis Manag 26(1):58-68

11. Ki E-J, Nekmat E (2014) Situational crisis communication and interactivity: usage and effectiveness of Facebook for crisis management by Fortune 500 companies. Comput Hum Behav 35:140-147

12. Schroeder A, Pennington-Gray L, Donohoe H, Kiousis S (2013) Using social media in times of crisis. J Travel Tour Mark 30(1-2):126-143 
Open Access This chapter is licensed under the terms of the Creative Commons Attribution 4.0 International License (http://creativecommons.org/licenses/by/4.0/), which permits use, sharing, adaptation, distribution and reproduction in any medium or format, as long as you give appropriate credit to the original author(s) and the source, provide a link to the Creative Commons license and indicate if changes were made.

The images or other third party material in this chapter are included in the chapter's Creative Commons license, unless indicated otherwise in a credit line to the material. If material is not included in the chapter's Creative Commons license and your intended use is not permitted by statutory regulation or exceeds the permitted use, you will need to obtain permission directly from the copyright holder.

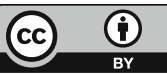

